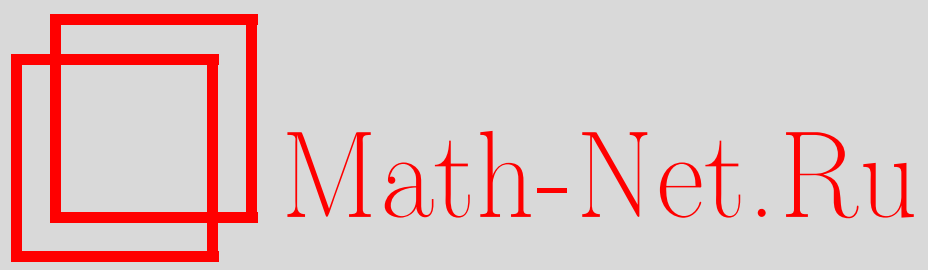

А. Черноуцан, Относительность движения в задачах динамики, Квант, 2019, номер 4, 40-46

DOI: https://doi.org/10.4213/kvant20190406

Использование Общероссийского математического портала Math-Net.Ru подразумевает, что вы прочитали и согласны с пользовательским соглашением http://www.mathnet.ru/rus/agreement

Параметры загрузки:

IP : 35.173 .219 .12

26 апреля 2023 г., 03:06:29

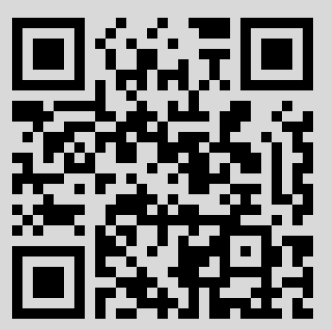




\section{ОтноСительнОСТЬ движения в задачах динамики}

\section{А. ЧЕРНОУЦАН}

АДАЧИ ДИНАМИКИ, В КОТОРЫХ УДОБно (а иногда и необходимо) использовать разные системы отсчета - CO, можно условно разделить на две группы. В задачах первой группы переход в другую СО является удобным приемом для учета кинематических связей, накладываемых на поведение двух движущихся тел нитями, стержнями, твердыми поверхностями (одно из тел движется по поверхности другого). В задачах второй группы дополнительная СО, например СО центра масс, используется для упрощения записи уравнений движения и законов сохранения импульса и энергии.

Поскольку мы будем использовать только поступательно движущиеся СО, то формулы сложения скоростей и ускорений выглядят совершенно одинаково:

$$
\begin{aligned}
& \vec{v}=\vec{v}_{\text {отн }}+\vec{v}_{\mathrm{CO}}, \\
& \vec{a}=\vec{a}_{\text {отн }}+\vec{a}_{\mathrm{CO}} .
\end{aligned}
$$

Приступим к рассмотрению конкретных задач.

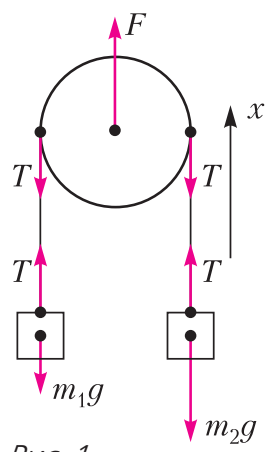

Задача 1. $К$ кониам нити, перекинутой через легкий блок, прикрепили грузы массами $m_{1}=3 \kappa 2$ и $m_{2}=10 \kappa 2$. К оси блока приложили силу, направленнуювертикально вверх и равную $F=120$ H (рис.1). С каким ускорением будет подниматься блок?

Решение. Поскольку блок идеальный (неве-

DOI: https://doi.org/10.4213/kvant20190406 сомый и вращается без трения), то силы натяжения нитей по обе стороны блока одинаковы (вращательный момент равен нулю). Второй закон Ньютона для невесомого блока имеет вид $F-2 T=0$, откуда находим $T=$ $=60 \mathrm{H}$. Записав уравнения второго закона Ньютона для грузов:

$$
\begin{aligned}
& T-m_{1} g=m_{1} a_{1 x}, \\
& T-m_{2} g=m_{2} a_{2 x},
\end{aligned}
$$

(ось $x$ направлена вверх), вычисляем $a_{1 x}=10 \mathrm{~m} / \mathrm{c}^{2}, a_{2 x}=-4 \mathrm{~m} / \mathrm{c}^{2}$. Чтобы найти ускорение блока, надо записать кинематическую связь между тремя ускорениями, обусловленную нерастяжимостью нити. Это можно сделать разными способами, например выразив длину нити через координаты грузов и блока и затем дважды продифференцировав это соотношение. Попробуйте проделать это самостоятельно. Мы же отметим, что в СО, связанной с блоком, кинематическая связь очевидна - относительные ускорения грузов равны по модулю и противоположны по знаку:

$$
\left(a_{16}\right)_{x}+\left(a_{26}\right)_{x}=0 .
$$

Используя формулу (2) (закон сложения ускорений), получаем

$$
\left(a_{1 x}-a_{6 x}\right)+\left(a_{2 x}-a_{6 x}\right)=0,
$$

откуда

$a_{6 x}=\frac{1}{2}\left(a_{1 x}+a_{2 x}\right)=\frac{F\left(m_{1}+m_{2}\right)}{4 m_{1} m_{2}}-g=3 \mathrm{~m} / \mathrm{c}^{2}$.

Задача 2. В иентре верхней грани вертикального иилиндра, стоящего на гладкой горизонтальной поверхности, сделали полусферическую выемку (рис.2). С края выемки съезжает маленькая шайба. Найди те силу давления шайбы на поверхность выемки в нижней точке движения. Масса шайбы $m_{1}=0,2 \kappa 2$, масса цилиндра $m_{2}=$ =0,4 к2. Трением пренебречь.

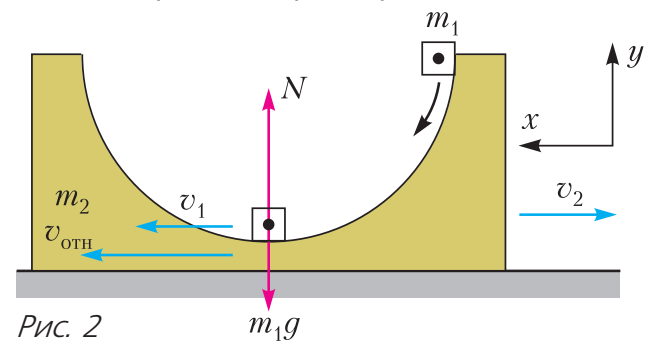


Решение. Искомая сила давления шайбы на поверхность выемки равна по величине силе реакции, которая входит во второй закон Ньютона, записанный для нижней точки траектории шайбы в проекции на вертикальную ось $y$. В СО земли траектория шайбы представляет собой сложную кривую (не окружность) за счет того, что цилиндр также движется, причем с переменной скоростью. Ускорение шайбы можно найти в СО, связанной с цилиндром, где шайба движется по окружности радиусом $R: a_{\text {отн }}=v_{\text {отн }}^{2} / R$. Поскольку ускорение цилиндра в проекции на ось $y$ в рассматриваемый момент равно нулю, то в соответствии с формулой (2) таким же будет ускорение шайбы относительно земли. Получаем

$$
N-m_{1} g=m_{1} \frac{v_{\text {отн }}^{2}}{R} .
$$

Закон сложения скоростей (1) в проекции на ось $x$ дает

$$
v_{\text {отн }}=v_{1}-\left(-v_{2}\right)=v_{1}+v_{2} .
$$

Запишем законы сохранения импульса и энергии:

$$
\begin{gathered}
0=m_{1} v_{1}-m_{2} v_{2}, \\
m_{1} g R=\frac{m_{1} v_{1}^{2}}{2}+\frac{m_{2} v_{2}^{2}}{2} .
\end{gathered}
$$

Из закона сложения скоростей и закона сохранения импульса выразим $v_{1}$ и $v_{2}$ через $v_{\text {отн }}$ :

$$
v_{1}=\frac{m_{2} v_{\text {отн }}}{m_{1}+m_{2}}, \quad v_{2}=\frac{m_{1} v_{\text {отн }}}{m_{1}+m_{2}}
$$

и подставим в закон сохранения энергии. Получим

$$
m_{1} g R=\frac{m_{1} m_{2}}{m_{1}+m_{2}} \frac{v_{\text {отн }}^{2}}{2},
$$

откуда выражаем $v_{\text {отн }}^{2}$, подставляем во второй закон Ньютона и находим силу реакции:

$$
N=m_{1} g+m_{1} \frac{v_{\text {отн }}^{2}}{R}=m_{1} g \frac{3 m_{2}+2 m_{1}}{m_{2}}=8 \mathrm{H} .
$$

Для проверки ответа можно $m_{2}$ устремить к бесконечности (неподвижный цилиндр) и мы получим известный результат $N=3 m_{1} g$.

Задача 3. Маленькая шайба массой $m_{1}$ соскальзывает с вершины гладкой полусферы радиусом $R$ и массой $m_{2}$, которая стоит на гладкой горизонтальной поверхности (рис.3). Найдите, для случая $m_{1}=m_{2}$, на

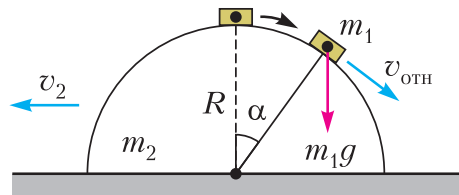

Рис. 3

какой высоте шайба оторвется от полусферы.

Решение. Поскольку в момент отрыва шайбы от поверхности полусферы ускорение полусферы обращается в ноль, то в соответствии с законом сложения ускорений (2) ускорение шайбы относительно земли равно ускорению шайбы относительно полусферы. В СО полусферы радиус кривизны траектории в момент отрыва все еще равен $R$ и проекция ускорения на радиальное направление равна $v_{\text {отн }}^{2} / R$. Второй закон Ньютона будет иметь вид

$$
m_{1} g \cos \alpha=m_{1} \frac{v_{\text {отн }}^{2}}{R} .
$$

Закон сложения скоростей (1) для данного случая

$$
\vec{v}_{1}=\vec{v}_{\text {отн }}+\vec{v}_{2}
$$

изображен на рисунке 4. С помощью теоремы косинусов выражаем $v_{1}$ :

$$
v_{1}^{2}=v_{2}^{2}+v_{\text {отн }}^{2}-2 v_{2} v_{\text {отн }} \cos \alpha .
$$

Закон сохранения импульса можно записать в СО земли (опираясь на рисунок 4), но проще всего сделать это в СО, движущейся влево со скоростью $v_{2}$ :

$$
\left(m_{1}+m_{2}\right) v_{2}=m_{1} v_{\text {отн }} \cos \alpha .
$$

Выразим отсюда $v_{2}$, подставим в закон сохранения энер-

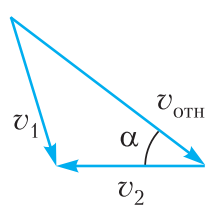

Pис. 4 гии

$$
m_{1} g R(1-\cos \alpha)=\frac{m_{1} v_{1}^{2}}{2}+\frac{m_{2} v_{2}^{2}}{2}
$$

и после преобразований придем к уравнению

$$
m_{1} g R(1-\cos \alpha)=\frac{m_{1} v_{\text {отн }}^{2}}{2}\left(1-\frac{m_{1} \cos ^{2} \alpha}{m_{1}+m_{2}}\right) .
$$

Подставляя $v_{\text {отн }}^{2}$ из второго закона Ньютона, приходим к кубическому уравнению относительно $\cos \alpha$ :

$$
\frac{m_{1}}{m_{1}+m_{2}} \cos ^{3} \alpha-3 \cos \alpha+2=0 .
$$

Проверкой правильности полученного урав- 
нения может служить то, что в пределе $m_{2} \rightarrow \infty$ (неподвижная полусфера) получается известный результат $\cos \alpha=2 / 3$. В случае $m_{1}=m_{2}$ уравнение

$$
x^{3}-6 x+4=0
$$

имеет простой корень $x=2$. Разделив кубический многочлен на $x-2$, придем к квадратному уравнению относительно $x=\cos \alpha$ :

$$
x^{2}+2 x-2=0 \text {, }
$$

откуда $\cos \alpha=\sqrt{3}-1$, а искомая высота отрыва шайбы

$$
h=R \cos \alpha=R(\sqrt{3}-1) .
$$

В следующих двух задачах ускорение СО не равно нулю и закон сложения ускорений работает в полную силу.

Задача 4. Маленькая шайба массой $m_{1}$ соскальзывает с вершины гладкого клина массой $m_{2}$, стоящего на гладкой горизонтальной плоскости (рис.5). Найдите вре-

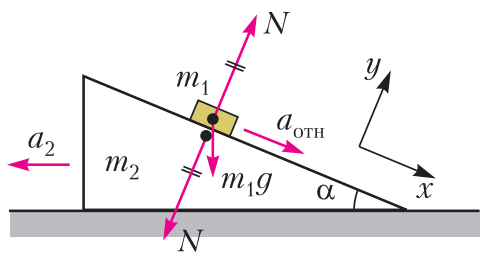

Pис. 5

мя соскальзывания шайбы, если высота клина $h$, а угол при основании клина $\alpha$.

Решение. Чтобы определить время соскальзывания шайбы, надо найти ускорение $a_{\text {отн }}$ шайбы относительно клина:

$$
\frac{h}{\sin \alpha}=\frac{a_{\text {отн }} t^{2}}{2} .
$$

Закон сложения ускорений (2) связывает ускорение $\vec{a}_{1}$ шайбы относительно земли,

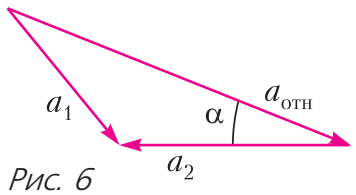
ускорение $\vec{a}_{2}$ клина (ускорение СО) и $\vec{a}_{\text {отн }}$ (рис.6):

$$
\vec{a}_{1}=\vec{a}_{\text {отн }}+\vec{a}_{2} .
$$

Опираясь на этот рисунок, запишем второй закон Ньютона для шайбы $\vec{N}+m_{1} \vec{g}=m_{1} \vec{a}_{1}$ в проекциях на оси $x$ и $y$ :

$$
\begin{gathered}
m_{1} g \sin \alpha=m_{1}\left(a_{\text {отн }}-a_{2} \cos \alpha\right), \\
N-m_{1} g \cos \alpha=-m_{2} a_{2} \sin \alpha .
\end{gathered}
$$

Проекция второго закона Ньютона для кли- на на горизонтальную ось имеет вид

$$
N \sin \alpha=m_{2} a_{2} .
$$

Исключая $N$ из последних двух уравнений, выражаем ускорение клина $a_{2}$ :

$$
a_{2}=\frac{m_{1} g \cos \alpha \sin \alpha}{m_{1} \sin ^{2} \alpha+m_{2}},
$$

после чего находим $a_{\text {отн }}$ :

$$
a_{\text {отн }}=g \sin \alpha \cdot \frac{m_{1}+m_{2}}{m_{1} \sin ^{2} \alpha+m_{2}} .
$$

(В пределе $m_{2} \rightarrow \infty$, неподвижный клин, получаем правильный ответ $g \sin \alpha$.) Подставив $a_{\text {отн }}$ в самое первое кинематическое равенство, найдем время соскальзывания.

Задача 5. Брусок стоит на гладкой горизонтальной плоскости, на бруске закреплен итатив, к которому на легкой нити подвешен груз массой $m_{1}$ (рис.7). Масса

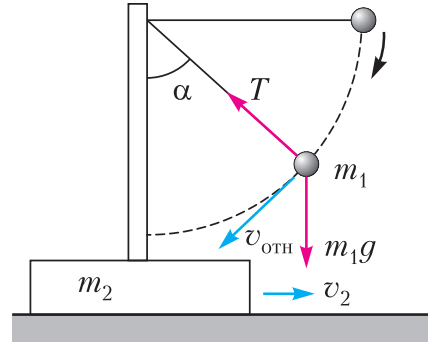

Pис. 7

бруска вместе со штативом равна $m_{2}$. Вначале нить с грузом удерживают в гори зонтальном положении, затем отпускают. Найдите силу натяжения нити в момент, когда нить составляет угол а с вертикалью.

Решение. Ускорение груза относительно земли выражается через ускорение бруска $\vec{a}_{2}$ и ускорение $\vec{a}_{\text {отн }}$ груза относительно СО, связанной с бруском (формула (2)):

$$
\vec{a}_{1}=\vec{a}_{\text {отн }}+\vec{a}_{2} .
$$

Это соотношение (точнее, два его «участника») изображено на рисунке 8 , где $\vec{a}_{\text {отн }}$ разложено на две составляющие - нормальную $\vec{a}_{n}$ и тангенциальную $\vec{a}_{\tau}$. Поскольку в СО бруска груз движется по окружности радиусом $l$, то $a_{n}=v_{\text {отн }}^{2} / l$. В проекции на ось, направленную вдоль нити к центру окружности, вто-

рой закон Ньютона для Рис. 8

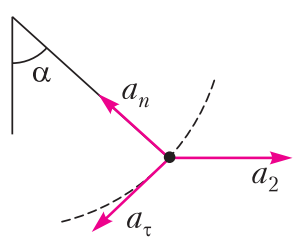


груза принимает вид

$$
T-m_{1} g \cos \alpha=\frac{m_{1} v_{\text {отн }}^{2}}{l}-m_{1} a_{2} \sin \alpha .
$$

Записав второй закон Ньютона для бруска в проекции на горизонтальную ось:

$$
T \sin \alpha=m_{2} a_{2}
$$

и исключив ускорение $a_{2}$, получим уравнение

$$
T\left(1+\frac{m_{1}}{m_{2}} \sin ^{2} \alpha\right)=\frac{m_{1} v_{\mathrm{OTH}}^{2}}{l}+m_{1} g \cos \alpha .
$$

Чтобы найти $v_{\text {отн }}^{2}$, надо записать законы сохранения импульса и энергии. Закон сохранения импульса проще всего записать в $\mathrm{CO}$, движущейся вправо со скоростью $v_{2}$ :

$$
\left(m_{1}+m_{2}\right) v_{2}=m_{1} v_{\text {отн }} \cos \alpha .
$$

Это соотношение мы используем, чтобы выразить $v_{2}$ через $v_{\text {отн }}$. А чтобы избавиться от $v_{1}^{2}$ в законе сохранения энергии

$$
m_{1} g l \cos \alpha=\frac{m_{1} v_{1}^{2}}{2}+\frac{m_{2} v_{2}^{2}}{2}
$$

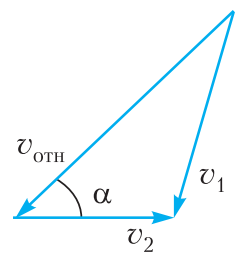

Рис. 9 запишем закон сложения скоростей (1):

$$
\vec{v}_{1}=\vec{v}_{\text {отн }}+\vec{v}_{2}
$$

изобразим его на рисунке 9 и воспользуемся теоремой косинусов:

$v_{1}^{2}=v_{2}^{2}+v_{\text {отн }}^{2}-2 v_{2} v_{\text {отн }} \cos \alpha$.

Подставив это выражение в закон сохранения энергии и выразив $v_{2}$ через $v_{\text {отн }}$, придем к уравнению

$$
m_{1} g l \cos \alpha=\frac{m_{1}}{2} \frac{m_{1} \sin ^{2} \alpha+m_{2}}{m_{1}+m_{2}} v_{\text {отн }}^{2} .
$$

Выразив отсюда $m_{1} v_{\text {отн }}^{2} / l$ и подставив в уравнение для $T$, получим

$$
T=\frac{m_{1} m_{2} g \cos \alpha}{m_{1} \sin ^{2} \alpha+m_{2}}\left(\frac{2\left(m_{1}+m_{2}\right)}{m_{1} \sin ^{2} \alpha+m_{2}}+1\right) .
$$

Полученный ответ может быть проверен двумя способами. При $m_{2} \rightarrow \infty$ (неподвижный брусок) это выражение переходит в известный результат $3 m_{1} g \cos \alpha$. При $\alpha=0$ это выражение совпадает с результатом задачи 2 .

Теперь рассмотрим несколько примеров того, как использование СО центра масс может существенно упростить задачу. Напомним, что координаты центра масс системы материальных точек вычисляются по формулам

$$
x_{ц}=\frac{m_{1} x_{1}+m_{2} x_{2}+\ldots}{m_{1}+m_{2}+\ldots}, \ldots,
$$

а скорость центра масс - по формуле

$$
\vec{v}_{\text {ц }}=\frac{m_{1} \vec{v}_{1}+m_{2} \vec{v}_{2}+\ldots}{m_{1}+m_{2}+\ldots}=\frac{\vec{p}_{\text {сист }}}{m_{\text {сист }}} .
$$

Ускорение центра масс равно отношению суммы всех внешних сил, действующих на тела системы, к массе системы:

$$
\vec{a}_{\text {ц }}=\frac{\vec{F}_{\text {внеш }}}{m_{\text {сист }}} .
$$

Из последних двух формул видно, что скорость центра масс замкнутой системы ( $\vec{F}_{\text {внеш }}=0, \quad \vec{p}_{\text {сист }}=$ const $)$ постоянна, т.е. $\mathrm{CO}$ центра масс замкнутой системы является инерциальной.

Задача 6. Шар массой $m$, движущийся со скоростью v, сталкивается с шаром массой $2 \mathrm{~m}$, движущимся ему навстречу со скоростью 2v. Найдите скорости шаров после удара, считая его иентральным и абсолютно упругим.

Решение. Задачу об упругом центральном ударе решают обычно через составление системы двух уравнений - законов сохранения импульса и энергии. Как правило, ограничиваются случаем, когда один из шаров вначале покоится, иначе система уравнений становится слишком громоздкой. Однако в СО центра масс конечные скорости определяются сразу, без составления системы уравнений. Импульс системы был и остается равным нулю, и достаточно изменить направление скоростей на противоположные, чтобы обеспечить и сохранение импульса и сохранение энергии.

Решение задачи состоит из нескольких шагов. Выберем положительное направление оси $x$ вдоль начальной скорости первого шара. Скорости в СО земли будем обозначать буквами $v$, относительно центра масс буквами $u$, скорости после удара будем писать со штрихом.

Шаг 1. Найдем скорость центра масс:

$$
v_{ц x}=\frac{m v-2 m \cdot 2 v}{m+2 m}=-v .
$$

ШІаг 2. Определим скорости шаров в СО центра масс до удара (формула (1)):

$$
\begin{gathered}
u_{1 x}=v_{1 x}-v_{\amalg x}=v-(-v)=2 v, \\
u_{2 x}=v_{2 x}-v_{\amalg x}=-2 v-(-v)=-v .
\end{gathered}
$$


Шаг 3 (главный). Определим скорости шаров в СО центра масс после удара:

$$
u_{1 x}^{\prime}=-u_{1 x}=-2 v, \quad u_{2 x}^{\prime}=-u_{2 x}=v .
$$

Шаг 4. Найдем конечные скорости шаров в изначальной СО с помощью закона сложения скоростей (1):

$$
\begin{gathered}
v_{1 x}^{\prime}=u_{1 x}^{\prime}+v_{\amalg x}=(-2 v)+(-v)=-3 v, \\
v_{2 x}^{\prime}=u_{2 x}^{\prime}+v_{\amalg x}=v+(-v)=0 .
\end{gathered}
$$

Задача 7. Шар массой $m_{1}$ налетает на покоящийся шар массой $m_{2}<m_{1}$. Найдите максимальный угол отклонения налетающего шара в результате косого абсолютно упругого удара. Поверхности шаров гладкие.

Решение. Перейдем в СО центра масс, скорость которого

$$
\vec{v}_{\text {แ }}=\frac{m_{1} \vec{v}_{1}}{m_{1}+m_{2}} .
$$

Начальные скорости шаров в этой СО равны

$$
\begin{aligned}
& \vec{u}_{1}=\vec{v}_{1}-\vec{v}_{\amalg}=\frac{m_{2} \vec{v}_{1}}{m_{1}+m_{2}}, \\
& \vec{u}_{2}=0-\vec{v}_{\amalg}=-\frac{m_{1} \vec{v}_{1}}{m_{1}+m_{2}} .
\end{aligned}
$$

Поскольку импульс системы после соударения должен остаться равным нулю, а энергия должна сохраниться, то конечные скорости шаров равны начальным, а направлены в противоположных направлениях (рис.10):

$$
u_{1}^{\prime}=u_{1}, \quad u_{2}^{\prime}=u_{2},
$$

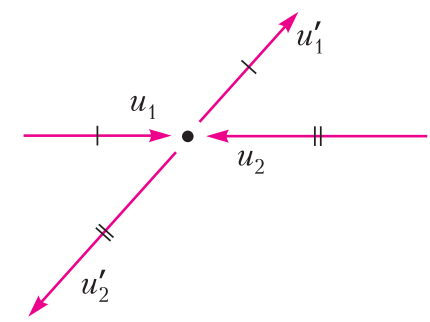

Рис. 10

причем $\overrightarrow{u_{1}^{\prime}}$ может составлять любой угол (от нуля до $\left.360^{\circ}\right)$ с $\vec{u}_{1}$. Возвращаясь в изначальную СО, получаем

$$
\overrightarrow{v_{1}^{\prime}}=\vec{v}_{\text {ц }}+\overrightarrow{u_{1}^{\prime}}
$$

Конец вектора $\overrightarrow{u_{1}^{\prime}}$ описывает окружность (рис.11). Максимальный угол между $\overrightarrow{v_{1}^{\prime}}$ и $\vec{v}_{1}$ соответствует касательной к окруж-

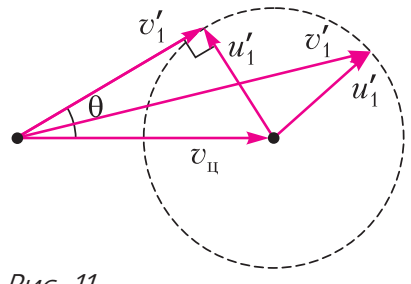

Pис. 11

ности:

$$
\sin \theta=\frac{u_{1}^{\prime}}{v_{\text {Ц }}}=\frac{m_{2}}{m_{1}} .
$$

Задача 8. Два шарика массами $m$ и 3m, соединенные нитью длиной $l$, движутся по гладкой горизонтальной поверхности. $B$ некоторый момент один из шариков (массой $m$ ) неподвижен, а скорость другого равна у инаправлена перпендикулярно нити. Чему равна сила натяжения нити?

Решение. В СО земли движение шариков выглядит сложно. Однако если перейти в $\mathrm{CO}$ центра масс, то каждый из шариков будет равномерно двигаться по окружности вокруг неподвижного центра масс. Центр масс находится между шариками на расстояниях

$$
l_{1}=\frac{m_{2} l}{m_{1}+m_{2}}=\frac{3}{4} l, \quad l_{2}=\frac{m_{1} l}{m_{1}+m_{2}}=\frac{1}{4} l
$$

от первого и второго шариков соответственно. Скорость центра масс относительно земли равна (рис.12)

$$
v_{\text {ц }}=\frac{m_{2} v}{m_{1}+m_{2}}=\frac{3}{4} v .
$$

Скорости шариков относительно центра масс

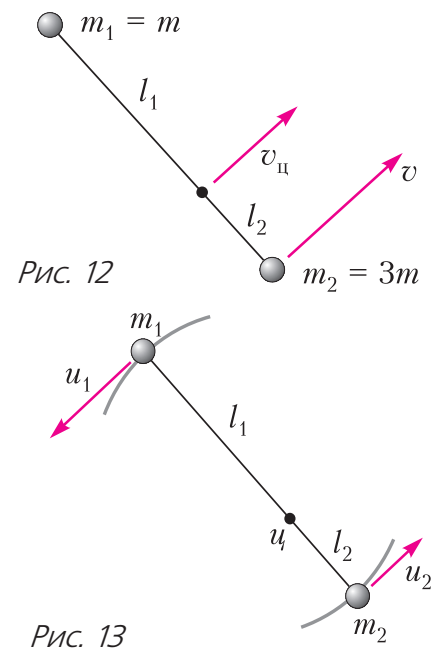


равны (рис.13)

$$
\begin{gathered}
u_{1}=0-v_{\text {ц }}=-\frac{m_{2} v}{m_{1}+m_{2}}=-\frac{3}{4} v, \\
u_{2}=v-v_{\text {ц }}=\frac{m_{1} v}{m_{1}+m_{2}}=\frac{1}{4} v .
\end{gathered}
$$

Сила натяжения нити может быть найдена из второго закона Ньютона для любого из шариков:

$$
T=\frac{m_{1} u_{1}^{2}}{l_{1}}=\frac{m_{2} u_{2}^{2}}{l_{2}}=\frac{m_{1} m_{2}}{m_{1}+m_{2}} \frac{v^{2}}{l}=\frac{3}{4} \frac{m v^{2}}{l} .
$$

В рассмотренных выше задачах мы обошлись без привлечения неинерциальных систем отсчета - НСО и сил инерции. Однако умение работать в НCO в некоторых задачах может быть незаменимым. Мы ограничимся несколькими примерами использования сил инерции в поступательно движущихся СО.

Выразим ускорение произвольного тела во втором законе Ньютона с помощью формулы сложения ускорений (2):

$$
\vec{F}=m\left(\vec{a}_{\text {отн }}+\vec{a}_{\mathrm{CO}}\right)
$$

( $\vec{F}$ - равнодействующая обычных сил) и перенесем $m \vec{a}_{\text {Со }}$ в левую часть равенства:

$$
\vec{F}+\left(-m \vec{a}_{\mathrm{CO}}\right)=m \vec{a}_{\text {отн }} .
$$

Определяя силу инерции формулой

$$
\vec{F}_{\text {ин }}=-m \vec{a}_{\mathrm{CO}},
$$

получаем второй закон Ньютона в $\mathrm{HCO}$ :

$$
\vec{F}+\vec{F}_{\text {ин }}=m \vec{a}_{\text {отн }} .
$$

Как и сила тяжести, сила инерции пропорциональна массе тела. Равнодействующая сил инерции, как и сил тяжести, приложена к центру тяжести тела. Во многих случаях оказывается удобным присоединить силу инерции к силе тяжести и считать, что появление сил инерции привело к изменению ускорения свободного падения:

$$
\overrightarrow{g^{\prime}}=\vec{g}-\vec{a}_{\mathrm{CO}}
$$

Задача 9. Во сколько раз изменится ииклическая частота колебаний математи ческого маятника, если точка подвеса бу дет перемещаться с горизонтальным ускорением $a=0,75 \mathrm{~g}$ ?

Решение. Перейдем в СО, связную с точкой подвеса маятника. В этой НСО действует горизонтальная сила инерции $\vec{F}_{\text {ин }}=-m \vec{a}$, где $\vec{a}$ - ускорение точки подвеса. В положе- нии равновесия нить будет направлена вдоль нового ускорения свободного падения $\overrightarrow{g^{\prime}}=\vec{g}-\vec{a}$ (рис.14), т.е. будет отклонена назад под углом, тангенс которого равен $a / g$. Циклическая частота малых колебаний маятника около этого поло-

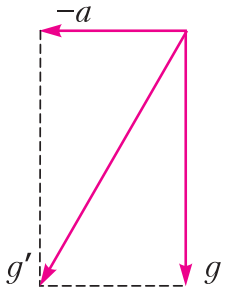

Pис. 14 жения равновесия равна

$$
\omega^{\prime}=\sqrt{\frac{g^{\prime}}{l}}=\sqrt{\frac{\sqrt{g^{2}+a^{2}}}{l}} .
$$

Отношение новой частоты колебаний к старой (с неподвижной точкой подвеса) равно

$$
\frac{\omega^{\prime}}{\omega}=\sqrt{\frac{g^{\prime}}{g}}=\sqrt{\frac{\sqrt{g^{2}+a^{2}}}{g}}=\sqrt{1,25} \approx 1,12 .
$$

Задача 10. Тело, плотность которого $\rho=500 \kappa 2 / \mathrm{m}^{3}$, плавает на поверхности воды в сосуде. Сосуд поднимается вертикально вверх с ускорением $a=g / 2$. Какая часть объема тела (в прочентах) погружена в воду?

Решение. Поскольку в уравнении

$$
\rho V g=\rho_{\text {в }} g V_{\text {погр }},
$$

выражающем условие плавания тела, ускорение $g$ сокращается, а переход в НСО, связанную с сосудом, сводится к замене $g$ на $g^{\prime}=g+a$, то доля погруженного объема не меняется:

$$
\frac{V_{\text {погр }}}{V}=\frac{\rho}{\rho_{\text {в }}}=\frac{1}{2}=50 \% .
$$

Решая эту задачу в СО земли, часто делают ошибку, полагая, что поскольку сила Архимеда возрастает, то глубина погружения должна увеличиться. Чтобы избежать этой ошибки, надо разобраться, как меняется формула для силы Архимеда за счет движения сосуда с ускорением. В НСО же такой проблемы нет.

Задача 11. Два одинаковых тела лежат на гладкой горизонтальной плоскости. Тела соединены прямой ненатянутой нитью длиной l. Середину нити начинают перемещать горизонтально перпендикулярно нити с ускорением а. Найдите, на сколько нагреются тела при ударе, если считать удар абсолютно неупругим, а удельную теплоемкость равной с. 
Решение. В СО земли движение тел выглядит сложно. Однако в СО, в которой центр нити неподвижен, на каждое из тел действует постоянная сила инерции $F_{\text {ин }}=m a$, направленная перпендикулярно нити. Такая задача эквивалентна задаче о падении и столкновении двух тел, связанных нитью, середина которой неподвижна, но с заменой $g$ на $a$. Закон сохранения энергии для такой задачи выглядит просто:

$$
2 \cdot m a \frac{l}{2}=c \cdot 2 m \cdot \Delta t, \text { откуда } \Delta t=\frac{a l}{2 c} .
$$

Напоследок - задача, в которой одно из действующих лиц имеет значительно большую массу, чем другое.

Задача 12. Санки съезжают с гладкой горки высотой $h$ и приобретают скорость v. Начальная потенциальная энергия $\mathrm{mgh}$ равна конечной кинетической энергии $m v^{2} / 2$. Однако в инерциальной CO, которая движется горизонтально со скоростью v, начальная энергия равна $m g h+m v^{2} / 2, a$ конечная равна нулю. Спасите закон сохранения энергии.

Решение. Чтобы понять, куда делась энергия, надо ввести в игру еще одного участника, энергия которого также будет меняться. Этим участником должно стать тело, с которым санки взаимодействуют при спуске. Этим телом является... земной шар! Оказывается, изменением энергии Земли (любого очень массивного тела) можно пренебречь только в СО, где это тело покоится. Изменение скорости Земли при скатывании санок можно найти из закона сохранения импульса

$$
m v-M \cdot \Delta V=0,
$$

и изменение энергии Земли, равное

$$
\frac{M(\Delta V)^{2}}{2}=\frac{m}{M} \frac{m v^{2}}{2},
$$

оказывается ничтожно малым. Однако в $\mathrm{CO}$, которая движется со скоростью $v$, изменение энергии Земли, равное

$$
\begin{aligned}
\frac{M(v+\Delta V)^{2}}{2}- & \frac{M v^{2}}{2}= \\
& =M v(\Delta V)+\frac{M(\Delta V)^{2}}{2} \approx m v^{2},
\end{aligned}
$$

не только не является бесконечно малым, но в точности компенсирует потерянную нами в условии энергию.
Мораль этой задачи такова: изменением энергии очень массивного тела можно пренебрегать только в СО, где это тело покоится. Хорошо знакомый пример: упругий удар мяча о стенку. Если стенка неподвижна, то мяч отскакивает с такой же скоростью, энергия стенки не меняется. Если же стенка движется, то скорость после отскока не равна начальной - энергия стенки изменяется!

\section{Упражнения}

1. К потолку кабины лифта, поднимающегося с ускорением $2 \mathrm{~m} / \mathrm{c}^{2}$, прикреплен динамометр. К динамометру подвешен блок, свободно вращающийся вокруг горизонтальной оси. Через блок перекинута нить, к концам которой прикреплены грузы массами 1 кг и 3 кг. Определите показания динамометра.

2. Демонстрационная установка состоит из наклонной плоскости, плавно переходящей в «мертвую петлю» радиусом $R$. Установка закреплена на тележке, стоящей на горизонтальной плоскости. Груз массой 0,2 кг съезжает с высоты $3 R$, отсчитанной от нижней точки петли. Чему равна сила давления груза в верхней точке петли? Трением пренебречь. Масса установки вместе с тележкой в 4 раза больше массы груза.

3. Два шара двигаются в одном направлении, первый со скоростью $v$, второй со скоростью $2 v$. Масса второго шара в 4 раза больше массы первого. Найдите скорости шаров после абсолютно упругого центрального удара.

4. В маятниковых часах используется математический маятник с периодом колебаний 1 c. Часы помещают в ракету, которая начинает подниматься с постоянным ускорением. Чему равно это ускорение, если за 7 с подъема маятник часов совершает 8 полных колебаний? Ускорение свободного падения $g=9,8 \mathrm{~m} / \mathrm{c}^{2}$.

5. Открытую цистерну в форме куба со стороной 2 м, стоящую на платформе, заполнили жидкостью наполовину. Платформа стала разгоняться с ускорением $2 \mathrm{~m} / \mathrm{c}^{2}$. На сколько поднялся уровень жидкости у задней стенки платформы к тому моменту, когда жидкость и платформа стали двигаться как единое целое?

6. Пуля массой 20 г, летящая со скоростью $100 \mathrm{~m} / \mathrm{c}$, попадает в летящий ей навстречу очень массивный шар, скорость которого $10 \mathrm{~m} / \mathrm{c}$, и застревает в нем. Какое количество теплоты выделилось при ударе?

7. Для системы, описанной в задаче 3 статьи, найдите зависимость силы давления $N$ шайбы от угла $\alpha$.

Указание. Воспользуйтесь подходом, развитом в задаче 5 статьи. 